\section{Loxoscelismo y cambios en el perfil de coagulación}

\section{Loxoscelism and changes in coagulation profile}

\section{Sr. Editor:}

El accidente por mordedura de arácnidos del género Loxosceles, es un problema de distribución mundial ${ }^{1}$, describiéndose panoramas epidemiológicos, como el publicado por Zavaleta y cols. en 1987 en Perú ${ }^{2}$. Clínicamente puede presentarse en uno de los dos siguientes cuadros, el cutáneo-visceral (sistémico) y cutáneonecrótico (cutáneo) $)^{3}$.

Conforme se han ido publicando comunicaciones de casos e investigaciones experimentales y observacionales, se ha puesto en vista, la relación entre el cuadro de loxoscelismo y cambios hemostáticos. Luna García y cols. ${ }^{4}$, reportaron el caso de una mujer de 19 años de edad, quien presentó un cuadro sistémico atípico, quien falleció posteriormente por una complicación, posiblemente por coagulación intravascular diseminada (CID). Dentro de los estudios realizados, se encontró una alteración del perfil de coagulación (trombocitopenia, prolongación del tiempo parcial de trombina y tromboplastina y descenso del fibrinógeno), con incremento del dímero $\mathrm{D}$ (D-D). Otro caso publicado por Rojas y cols. ${ }^{5}$, es el de una mujer de 62 años, quien sólo presentó un loxoscelismo cutáneo sobreinfectado, en que se encontró D-D y fibrinógeno elevado. Tavares y cols. ${ }^{6}$, estudiaron los cambios bioquímicos, hemostáticos y hematológicos en modelos experimentales animales, inyectados con veneno de Loxosceles gaucho. Tomaron análisis de los mismos a las tres, 24, 48, 72 y $120 \mathrm{~h}$, posterior a la exposición. Las primeras alteraciones hemostáticas fueron la trombocitopenia (a las tres y $24 \mathrm{~h}$ ), seguida de trombocitos (a las 72 y $120 \mathrm{~h}$ ), hiperagregación plaquetaria (a las 48 y $72 \mathrm{~h}$ ), con elevación del fibrinógeno todo el tiempo. Los factores V, VII, VIII, IX, X and XI se incrementaron a las 120 horas. No se reportó CID. Mac Glasson y cols. ${ }^{3}$, usando modelos animales, en un experimento similar al de Tavares, estudió el efecto del veneno en la coagulación y en la piel. Recolectó y analizó muestras a las 24,48 y $72 \mathrm{~h}$. A las $24 \mathrm{~h}$, se observó trombocitopenia y descenso de la proteína C. Posteriormente hubo prolongación de tiempo parcial de tromboplastina activada, incremento del fibrinógeno y los factores V, VII, VIII, IX, X. No reportó CID. Malaque y cols. ${ }^{7}$, analizaron 81 pacientes diagnosticados con loxoscelismo cutáneo o sistémico después de una o dos semanas del accidente. Se encontró altos niveles de D-D, pero trombocitopenia sólo en 17,6\% en la primera semana.
El cambio en el perfil de coagulación, está más relacionado al problema necrótico, donde las lesiones cutáneas presentan en su histopatología, presencia de vasculitis y trombosis ${ }^{3,6}$. En estos cambios, se observa una activación de la cascada de coagulación y agregación plaquetaria, lo que explica los cambios en el perfil, especialmente en el número de plaquetas. Clínicamente, la alteración de la coagulación y el hallazgo relacionado, como el D-D, puede observarse tanto en casos complicados o no, lo que posiblemente lo descartaría como un factor predictor de gravedad. La alteración en el número de plaquetas, el D-D y el incremento de factores de coagulación, deben ser investigadas para determinar, si alguno tiene importancia clínica en el futuro. Además, si estos cambios se asocian con otras alteraciones como el cumplimiento de criterios para CID, fiebre, hemólisis u otro factor que indique complicaciones. En ese caso la intervención agresiva está indicada.

\section{Referencias bibliográficas}

1.- Schenone H, Saavedra T, Rojas A, Villarroel F. Loxoscelism in Chile: epidemiological, clinical and experimental studies. Rev Inst Med Trop São Paulo 1989; 31: 403-15.

2.- Zavaleta A. Loxoscelismo, un problema de salud en el Perú. Bol Of Sanit Panam. 1987;103: 378-86.

3.- McGlasson D L, Harroff H H, Sutton J, Dick E, Elston D M. Cutaneous and systemic effects of varying doses of brown recluse spider venom in a rabbit model. Clin Lab Sci 2007; 20: 99-105.

4.- Luna García L, Ugarte Briones C, Soto Ma. Elena , Ávila Casado Ma. del Carmen. Picadura por arácnido. Rev Fac Med UNAM. 2000; 43: 101-4.

5.- Rojas Reyna G, Silva Velazco J, Sarue Saed N, Hernández Vera F, Baquera Heredia J. Necrosis cutánea por loxoscelismo. Reporte de caso. Rev Mex Angiol 2009; 37 : 93-6.

6.- Tavares F L, Sousa-e-Silva M C, Santoro M L, Barbaro K C, Rebecchi I M, Sano-Martins I S. Changes in hematological, hemostatic and biochemical parameters induced experimentally in rabbits by Loxosceles gaucho spider venom. Hum Exp Toxicol 2004; 23: 477-86.

7.- Malaque C M, Santoro M L, Cardoso J L, Conde M R, Novaes C T, Risk JY, et al. Clinical picture and laboratorial evaluation in human loxoscelism. Toxicon 2011; 58: 664-71.

Rafael Pichardo-Rodríguez, J. Antonio Grandez-Urbina Universidad Ricardo Palma. Lima, Perú.

Correspondencia a: Rafael Pichardo-Rodríguez rafael_martin1352@hotmail.com 